March 2010

\title{
The utility of modified Butler-Leggett criteria for right ventricular hypertrophy in detection of clinically significant shunt ratio in ostium secundum-type atrial septal defect in adults
}

\author{
Adeel M. Siddiqui \\ Aga Khan University \\ Zainab Samad \\ Nina Hakacova \\ James Kinsella \\ CaryWard
}

See next page for additional authors

Follow this and additional works at: https://ecommons.aku.edu/pakistan_fhs_mc_med_cardiol Part of the Cardiology Commons

\section{Recommended Citation}

Siddiqui, A., Samad, Z., Hakacova, N., Kinsella, J., Ward, C., White, M., Crowley, A., Wagner, G., Harrison, J. (2010). The utility of modified Butler-Leggett criteria for right ventricular hypertrophy in detection of clinically significant shunt ratio in ostium secundumtype atrial septal defect in adults. Journal of Electrocardiology, 43(2), 161-166.

Available at: https://ecommons.aku.edu/pakistan_fhs_mc_med_cardiol/36 
Authors

Adeel M. Siddiqui, Zainab Samad, Nina Hakacova, James Kinsella, Cary Ward, Michael White, Anna Lisa C. Crowley, Galen S. Wagner, and J. Kevin Harrison 


\title{
The utility of modified Butler-Leggett criteria for right ventricular hypertrophy in detection of clinically significant shunt ratio in ostium secundum-type atrial septal defect in adults
}

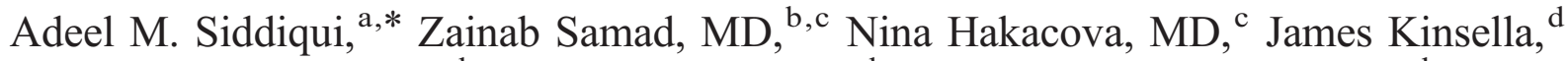 \\ Cary Ward, MD, ${ }^{\mathrm{b}}$ Michael White, MD, ${ }^{\mathrm{b}}$ Anna Lisa C. Crowley, MD, ${ }^{\mathrm{b}}$ \\ Galen S. Wagner, MD, ${ }^{\mathrm{b}, \mathrm{c}}$ J. Kevin Harrison, $\mathrm{MD}^{\mathrm{b}}$ \\ ${ }^{\mathrm{a}}$ The Aga Khan University Hospital, Karachi, Pakistan \\ ${ }^{\mathrm{b}}$ Division of Cardiology, Department of Medicine, Duke University Medical Center, Durham, NC, USA \\ ${ }^{\mathrm{c}}$ Duke Clinical Research Institute, Durham, NC, USA \\ ${ }^{\mathrm{d}}$ University of Glasgow, Glasgow, United Kingdom
}

Received 8 June 2009

\begin{abstract}
Background: This study was performed to test the hypothesis that there exists a correlation between the Butler-Leggett $(\mathrm{BL})$ criterion for right ventricular hypertrophy on the electrocardiogram and the Qp/Qs shunt ratio in adults with ostium secundum atrial septal defects (ASDs).

Methods: Demographic, cardiac catheterization, ASD closure, and electrocardiographic data were acquired on 70 patients with secundum ASDs closed percutaneously. Simple linear regression and logistic regression models were created to test the hypothesis.

Results: The mean $\mathrm{Qp} / \mathrm{Qs}$ ratio and $\mathrm{BL}$ criterion value were $1.61 \pm 0.46$ and $0.11 \pm 0.41$, respectively. The $\mathrm{BL}$ criterion values correlated with shunt ratios $\left(r^{2}=0.11\right.$ and $\left.P=.004\right)$. A BL criterion value greater than $0 \mathrm{mV}$ predicted a significant shunt ratio ( $\mathrm{Qp} / \mathrm{Qs} \geq 1.5$ ) (odds ratio, 4.8; $95 \%$ confidence interval, $1.3,18.1 ; P=<.0001$ ) with a sensitivity of 0.68 and specificity of 0.65 . Conclusion: Our results indicate that there is limited utility of the BL criterion at detecting right ventricular volume overload, although a BL criterion value greater than $0 \mathrm{mV}$ being used to identify patients with significant intracardiac shunts yielded a sensitivity of 0.68 and specificity of 0.65 .
\end{abstract} (C) 2010 Elsevier Inc. All rights reserved.

Keywords: $\quad$ Butler-Leggett criteria; Atrial septal defect; Right ventricular volume overload

\section{Introduction}

Atrial septal defects (ASDs) account for $5 \%$ to $10 \%$ of congenital heart defects, and $75 \%$ of this population are of the ostium secundum type. ${ }^{1}$ Untreated, ASDs can lead to impaired aerobic capacity, overt congestive heart failure, atrial arrhythmias, and pulmonary hypertension. ${ }^{2,3}$ Echocardiography is commonly used to diagnose ASDs, whereas cardiac magnetic resonance imaging and/or transesophageal echo may be used to further define the ASD anatomy and shunt magnitude. ${ }^{4,5}$ In addition, cardiac catheterization provides direct assessment of intracardiac and intrapulmonary hemodynamics as well as oximetric calculation of the magnitude of the intracardiac shunt. These methods are

* Corresponding author. The Aga Khan University Hospital, 46-A 2/2 3rd Sunset Street, Phase 2 Extension, Defence Housing Authority, Karachi, Pakistan.

E-mail address: adeelmsiddiqui@gmail.com excellent for evaluating patients with ASDs; however, cardiac catheterization is invasive and thus carries the risk of serious complications. ${ }^{6}$ Magnetic resonance imaging, although noninvasive, is contraindicated in some patients and is not widely available. ${ }^{7}$

The standard electrocardiogram (ECG), a quick, low-cost procedure that is usually performed in the routine evaluation of a cardiac patient, can possibly provide clues regarding hemodynamics. Many ECG changes such as right axis deviation, increased PR interval, and right atrial enlargement have been described in patients with ASDs. ${ }^{8}$ Therefore, the potential clinical value of using the inexpensive and readily available standard ECG to assess hemodynamic status and physiologic impact of the intracardiac shunt in individuals with ostium secundum ASDs should be determined.

The Butler-Leggett (BL) electrocardiographic criterion is a quantitative tool developed to evaluate intracardiac, specifically right ventricular, pressure overload. ${ }^{9}$ The BL 
criterion has been shown to estimate the amount of right ventricular hypertrophy (RVH) in patients with mitral stenosis using a standard 12-lead ECG. ${ }^{9}$ The amplitudes of ECG waveform in leads $I, V_{1}, V_{2}$, and $V_{6}$ are used to calculate a value that can estimate the degree of RVH. This value gives an estimate of the amount of net electrical activity in the heart reflecting the balance of currents in the right and left sides of the heart. ${ }^{9}$ The right ventricle progressively enlarges when compensating for either a pressure or volume overload; however, this "enlargement" is in the form of hypertrophy with increased pressure and dilation with increased volume. It has been previously established that pressure overload (hypertrophy) and volume overload (dilation) produce different changes on the ECG. ${ }^{10}$ To date, the BL criterion has not been tested to determine its value in conditions of right ventricular (RV) volume overload. ${ }^{9}$

This study was performed to determine whether BL ECG measurements correlate with hemodynamic measurements and the magnitude of the intracardiac shunt $(\mathrm{Qp} / \mathrm{Qs}$ ratio) in adults with RV volume overload due to ostium secundum ASDs.

\section{Methods}

\section{Population}

Subjects were consecutive patients ( $>18$ years old) with ostium secundum ASDs who underwent cardiac catheterization and ASD closure at Duke University Medical Center between March 2002 to December $2007(\mathrm{n}=131)$. Patients were excluded if they lacked an available ECG within 6 months before closure $(n=48)$ or if the ECG revealed a complete right bundle-branch block ${ }^{11}(\mathrm{n}=9)$, atrial fibrillation $(\mathrm{n}=2)$, or an implanted pacemaker $(\mathrm{n}=2)$. Thus, 70 adult patients were examined in the study.

\section{Cardiac catheterization}

All cardiac catheterization procedures were performed in the Duke University Medical Cardiac Catheterization Laboratories using a standardized protocol for evaluation of ASD patients. Cardiac catheterization was performed via the femoral vessels using local anesthesia and sedation. General anesthesia was not used. Hemodynamic data were obtained using a balloon floatation catheter (Medtronic Inc, Minneapolis, MN) connected to a fluid-filled pressure transducer. In each patient, pressure measurements from the right atrium (RA), the RV, the pulmonary artery (PA), the left atrium, and the femoral artery were obtained. In addition, oximetric measurements (AVOXimeter 1000E; ITC, Piscataway, NJ) were obtained from the innominate vein, the high superior vena cava (SVC) cephalad to the right main stem bronchus, the low SVC caudal to the right main stem bronchus, the inferior vena cava, the RA, the RV, the PA, the left upper lobe pulmonary vein, and the femoral artery. These oximetric data were used to calculate intracardiac blood flow using the Fick principle. Oxygen consumption was calculated based on age, sex, and body surface area measurements. Pulmonary blood flow (Qp) and systemic blood flow (Qs) were calculated from the oxygen consumption divided by the difference of pulmonary venous, pulmonary arterial, systemic arterial, and systemic mixed venous oxygen content as appropriate. The systemic mixed venous oxygen level was calculated from the equation $(3 * \mathrm{SVC}+$ inferior vena cava) $/ 4$. The low $\mathrm{SVC}$ saturation was used as SVC in the absence of anomalous pulmonary venous return. The shunt ratio was determined as $\mathrm{Qp} / \mathrm{Qs}$.

Hemodynamic, demographic, and risk factor data was extracted electronically from the Duke Information System for Cardiovascular Care (DISCC) database at Duke University Medical Center.

\section{ECG interpretation}

Standard 12-lead ECGs were printed from the Philips TraceMasterVue, (Bothell, WA), (2006) database at Duke University Medical Center and analyzed on each patient by the investigators as described below. On all ECGs, we collected BL criterion values ${ }^{9}$ (see below), $\mathrm{P}$ wave duration and amplitude in leads II and $\mathrm{V}_{1}$, QRS duration, PR interval, and QRS axis. All wave amplitudes were measured to the nearest $0.05 \mathrm{mV}$. P wave amplitude was measured starting from the baseline of the $\mathrm{P}$ wave, with baseline defined at the level of PR segment. P wave duration was measured from beginning of $\mathrm{P}$ wave to point where wave reaches baseline. QRS duration and QRS axis were read directly off ECG software (Philips TraceMasterVue 2006). A modified version of the original $\mathrm{BL}$ criterion $^{9}$ was used for this study. In this version, let $A=$ the largest positive QRS deflection in $\mathrm{I}$ or $\mathrm{V}_{6}, R=$ absolute value of the largest negative deflection in $\mathrm{I}_{\text {or }} \mathrm{V}_{6}$, and $\mathrm{PL}=$ absolute value of the largest negative QRS deflection in V1. Afterward, the amplitude score $=A+R-\mathrm{PL}$ (in the original $\mathrm{BL}$ criteria, only the $\mathrm{S}$ wave amplitude in $\mathrm{V}_{1}$ was included in the $R$ component; and only the $\mathrm{Q}$ wave amplitude was included in the PL component.) When referring to the BL criterion, we will be referring to the modified version used for this study. The method for measuring $A, R$, and PL to calculate the modified BL value is detailed in Fig. 1.

\section{Statistical data analysis}

A descriptive analysis of the data set was first performed to determine the distribution of age, sex, comorbidities, ECG abnormalities, and hemodynamic variables. Continuous variables are described by calculated means, medians, and standard deviations. Categorical variables were described as percentages.

Once the normality assumption was met, simple linear regression models were created to examine the unadjusted association of increasing $\mathrm{BL}$ criterion values with $\mathrm{Qp} / \mathrm{Qs}$ ratios and mean PA pressures. Patients with complete right bundle-branch blocks excluded from the above analysis were later included in the analysis to examine the association of PR interval and $\mathrm{QRS}$ duration with $\mathrm{Qp} / \mathrm{Qs}$ ratio. To determine the predictive value of the BL criterion for a significant shunt, we created a logistic regression model where $\mathrm{Qp} / \mathrm{Qs}$ ratio of at least 1.5 was deemed clinically significant. Although a BL criterion value of at least $0.7 \mathrm{mV}$ has been used to define 


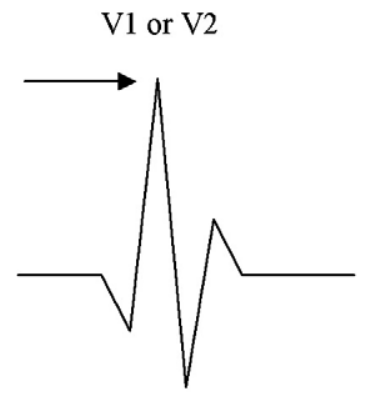

$\mathrm{A}=$ largest amplitude

of positive deflection
Lead I or V6

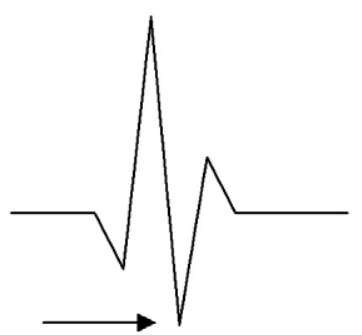

$\mathrm{R}=$ absolute value of the largest amplitude of negative deflection
V1

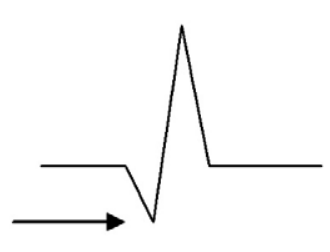

$\mathrm{PL}=$ absolute value of the largest amplitude of negative deflection

Fig. 1. Waveforms to be measured using modified BL criterion. $\rightarrow=$ waveform to be measured.

$\mathrm{RVH}$, given different changes with RV volume overload, we chose to test the diagnostic performance of different values: greater than or equal to $-0.95 \mathrm{mV}$ to greater than or equal to $1.0 \mathrm{mV}$, in predicting a significant ratio.

To further investigate whether the $\mathrm{BL}$ criterion could predict a significant shunt ratio, that is, $\mathrm{Qp} / \mathrm{Qs}$ of at least 1.5, we constructed a logistic regression model where a significant shunt ratio was treated as a dichotomous variable. The odds ratio in this case is an effect size measure, preferred in binary logistic regression modeling when comparing predictor variables. In this example, for a continuous predictor like the BL criterion, the log odds of the dependent variable (significant shunt ratio) changes when the continuous independent variable changes by 1 unit.

Two-sided $P$ values were used for all analyses, and the level of statistical significance was set a priori at .05 . All statistical analyses were performed using SAS E-Guide version 4.1 for Windows (SAS Institute, Cary, NC).

\section{Quality control}

Three investigators (JKH, CW, and MW) conducted the cardiac catheterizations and performed hemodynamic calculations. These data were recorded in the DISCC and accessed retrospectively for the purposes of this study. Two independent investigators (AMS and ZS) blinded to patient identifiers and catheterization data analyzed the ECGs on each patient. Each reading was subsequently compared for disagreements, which was defined as any difference in amplitude greater than $0.1 \mathrm{mV}$ or any difference in duration greater than 40 milliseconds. Any disagreements were settled by a third independent investigator (GSW). In addition, the third investigator (GSW) quality controlled QRS durations by comparing software interpretations to manually determined QRS durations in each ECG.

\section{Results}

In the population, $47(67 \%)$ were women, with a mean age of $51 \pm 19$ years. The mean size of the ASD closure device was $20 \mathrm{~mm}$, with a range of 8 to $36 \mathrm{~mm}$. Thirteen (19\%) had symptoms of heart failure prior to presentation, 4
(6\%) had diabetes mellitus, 22 (31\%) had hypertension, 11 $(16 \%)$ had dyslipidemia, and 1 (1\%) had a history of coronary artery disease (Table 1 ).

Hemodynamic measurements relating to both RV volume and pressure overload are presented in Table 1. The presence of RV volume overload was documented by the Qp/Qs shunt ratio: the mean was $1.63 \pm 0.46$, with a range of 0.88 to 2.99 . The absence of RV pressure overload was documented by the RV and PA pressures (Table 1). The means of RV systolic pressure and mean PA pressure were $37 \pm 13$ and $22 \pm 9 \mathrm{~mm}$ $\mathrm{Hg}$, respectively. Most patients in the study (67\%) had PA mean pressures less than $25 \mathrm{~mm} \mathrm{Hg}$, which is the upper limit of normal.

The ECG data showed a mean BL criterion value of $0.11 \pm$ $0.41 \mathrm{mV}$, thus far less than the value established for diagnosis of $\mathrm{RVH}$ of $0.7 \mathrm{mV}$. The values ranged from -0.95 to $1.0 \mathrm{mV}$, and only 4 patients $(6 \%)$ had $\mathrm{BL}$ values $>0.7 \mathrm{mV}$.

The BL criterion values between -1.0 and $1.0 \mathrm{mV}$ were normally distributed (verified using a quantile-quantile plot). The median BL criterion value was $0.10 \mathrm{mV}$.

The correlation between BL criterion values and the $\mathrm{Qp} / \mathrm{Qs}$ shunt ratio is shown in Fig. 2. There is a statistically significant correlation $\left(r^{2}=0.11\right.$ and $P=.004 ; 95 \%$ confidence interval, 0.12-0.64), indicating that the amplitude score explained $11 \%$ of the total variability of the shunt ratio.

After constructing the logistic regression model described above, we found that the odds of a significant shunt ratio increased by a factor of 4.8 (95\% confidence interval, 1.3-18.1) with every unit increase in the BL criterion. Every unit refers to $1 \mathrm{mV}(c=0.67) ; c$ refers to the c statistic, which is a measure of the discriminative power of the logistic equation.

A receiver operating characteristic (ROC) curve is shown in Fig. 3; the point closest to the upper left corner of the curve represents the optimal threshold $\mathrm{BL}$ criterion value $(>0 \mathrm{mV})$ that yields the optimal sensitivity of 0.68 , specificity of 0.65 , positive predictive value of 0.77 , and negative predictive value of 0.55 for detecting a shunt ratio $>1.5$. In addition, we also calculated likelihood ratios to enhance the interpretive value of this test. The likelihood ratio is 1.94 for a positive test and is 0.49 for a negative test. 
Table 1

Baseline characteristics of all patients

\begin{tabular}{ll}
\hline Demographic data & Patients $(\mathrm{n}=70)$ \\
\hline Men (\%) & $23(33 \%)$ \\
Age at time of closure (y) & $51 \pm 15(23-73)$ \\
Male & $51 \pm 19(18-84)$ \\
Female & $169 \pm 58(93-526)$ \\
Weight (lb) & $65.3 \pm 4.2(58-76)$ \\
Height (in) & \\
& \\
Risk factors & $4(6 \%)$ \\
Cerebrovascular disease & $11(16 \%)$ \\
Hyperlipidemia & $22(31 \%)$ \\
Hypertension & $4(6 \%)$ \\
Diabetes &
\end{tabular}

Cardiac catheterization data

RA pressure (mm Hg)

RV systolic pressure $(\mathrm{mm} \mathrm{Hg})$

Mean PA pressure (mm $\mathrm{Hg}$ )

Pulmonary blood flow (Qp, L/min)

System blood flow (Qs, L/min)

Qp/Qs shunt ratio

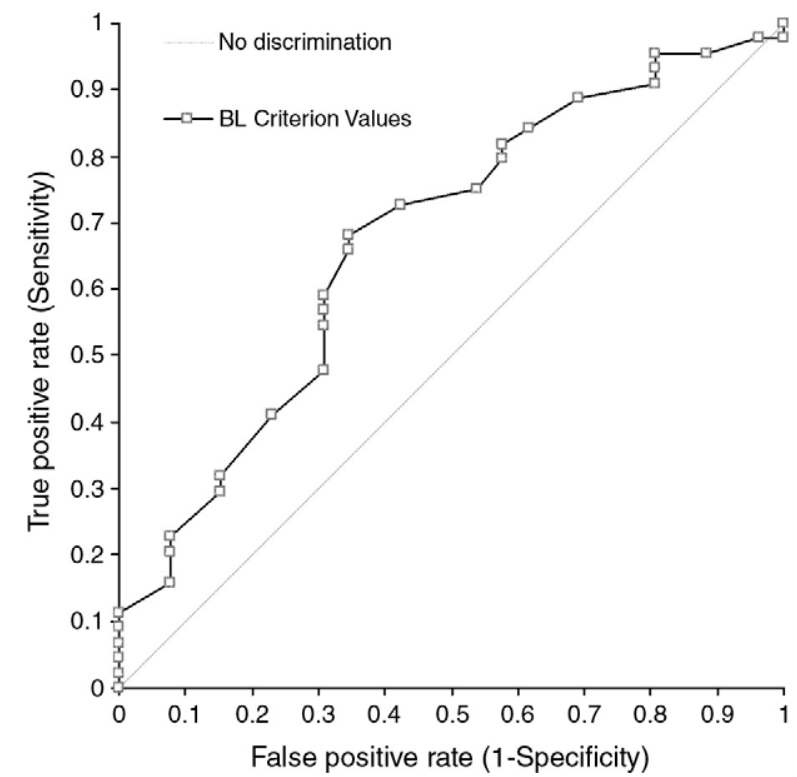

Fig. 3. The ROC curve with true positives (sensitivity plotted against false positives [ 1 - specificity]) for stepwise decreases of the threshold for the BL

was compensated by increasing RV pressures, as picked up by the BL criterion. We found that there was a weak correlation between $\mathrm{BL}$ criterion values and PA mean pressure $\left(r^{2}=0.08\right.$ and $\left.P=.01\right)$, with the $\mathrm{BL}$ criterion accounting for $8 \%$ of the mean PA pressure variance.

The relationship of QRS duration and PR interval was considered because of previous studies indicating increased prevalence of right bundle-branch block and increased PR interval in patients with ASDs. ${ }^{8}$ We found no correlation between the QRS duration and $\mathrm{Qp} / \mathrm{Q}$ s shunt ratio $\left(r^{2}=0.01\right.$, $P=.36)$. The relationship between $\mathrm{PR}$ interval and the $\mathrm{Qp} / \mathrm{Qs}$ shunt ratio was statistically significant, demonstrating a weak positive correlation $\left(r^{2}=0.09, P=.01\right)$.

Finally, in a multivariable model including PR interval and QRS duration, the BL criterion was an independent predictor of an increasing shunt ratio (adjusted $r^{2}=0.17, P=.002$ ).

\title{
Discussion
}

To our knowledge, this is the first study evaluating ECG $\mathrm{BL}$ criterion for $\mathrm{RVH}$ and traditionally reported $\mathrm{ECG}$ abnormalities in adult patients with ostium secundum ASDs. Despite the development of the BL ECG criterion to predict RV hypertrophy, our findings demonstrate a role of the BL criterion in predicting significant shunt ratios in patients with RV enlargement and volume overload due to ostium secundum ASDs.

The subject population of catheterization-based ASD closures was selected because this population allowed us to study the possible correlation of the ECG and the

Fig. 2. Scatter diagram for shunt ratios plotted against BL criterion score values with least squares regression line. The $R^{2}$ value of 0.11 indicates that $11 \%$ of the shunt ratio variance could be accounted for by the BL criterion score.

\begin{abstract}
$0.36 \pm 0.24(0.05-1.2) \quad$ criterion score values. Note that the maximum sensitivity of $68 \%$ and the
$\begin{array}{ll}0.11 \pm 0.41(-0.95 \text { to } 1.0) & \text { criterion score values. Note that the maximum sensitivity of } 68 \% \text { and the } \\ 0.29 \pm 0.18(0-05-1.2) & \text { maximum specificity of } 65 \% \text { are obtained for the amplitude score greater }\end{array}$ than $0 \mathrm{mV}$ (corresponding to the point of the curve closest to the upper lefthand corner of the figure)

$\begin{array}{ll}0.11 \pm 0.41(-0.95 \text { to } 1.0) & \text { criterion score values. Note that the maximum sensitivity of } 68 \% \text { and the } \\ 0.36 \pm 0.24(0.05-1.2) & \text { maximum specificity of } 65 \% \text { are obtained for the amplitude score greater }\end{array}$

$\begin{array}{ll}0.11 \pm 0.41(-0.95 \text { to } 1.0) & \text { criterion score values. Note that the maximum sensitivity of } 68 \% \text { and the } \\ 0.36 \pm 0.24(0.05-1.2) & \text { maximum specificity of } 65 \% \text { are obtained for the amplitude score greater }\end{array}$

$0.54 \pm 0.28(0-1.2)$
\end{abstract}

$0.08+0.04(0.0-0.2)$

$17(24 \%)$ $20.3 \pm 6.06(8-36)$

chance of a sionificant shunt ratio; however, a negative value cannot rule out a significant shunt and does not yield We analyzed the correlation between BL criterion values

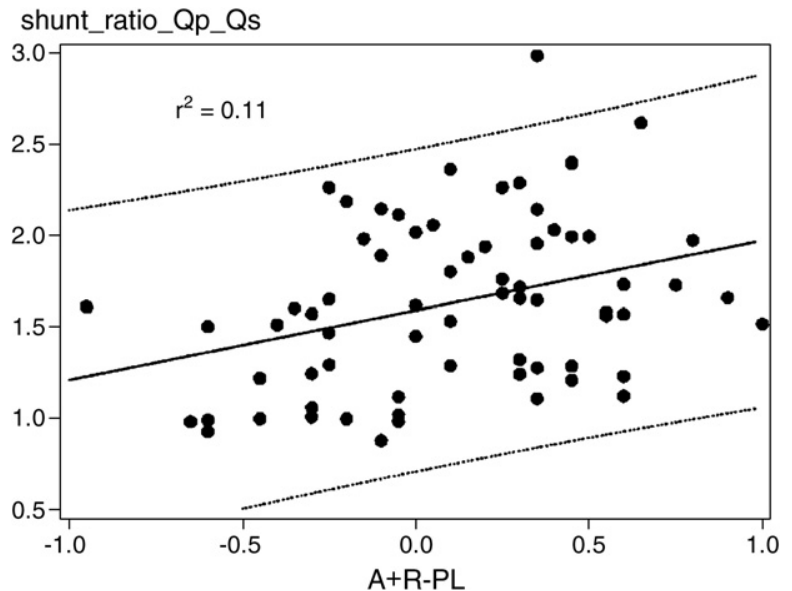$$
\text { criterion score. }
$$ 
$\mathrm{Qp} / \mathrm{Qs}$ shunt ratio, with both data points being prior to catheterization-based ASD closure. The Amplatzer septal occluder (AGA Medical, Plymouth, MN) and Helex septal occluder (Gore Medical, Flagstaff, AZ), being the most widely used devices with proven advantages, were included in the study population. ${ }^{12,13}$ Because ECG changes such as reduction in the QRS duration and PR interval suggestive of decreased volume overload are seen post-ASD closure, it was imperative that all ECGs on patients included in our study be prior to closure of the ASD. ${ }^{14}$

The original BL criterion for the conventional 12-lead ECG was formulated by considering the projections of the horizontal plane QRS loop on 3 directions: anterior, right, and posterior-left. As in the BL criterion, $A$ refers to the anterior component, $R$ to the rightward component, and $P L$ to the posterior-left component. As already noted in the ECG methods section, in the original BL criterion, only the $\mathrm{S}$ wave amplitude in $\mathrm{V}_{1}$ was included in the $R$ component; and only the $\mathrm{Q}$ wave amplitude was included in the PL component. In our new amplitude score, the sum $A+R$ approximates the voltage in the anterior-right direction; and PL, the posteriorleft direction. Thus, the score $(A+R-\mathrm{PL})$ can be considered to reflect the net balance of electrical activity in direction from posterior-left to anterior-right. Other RV hypertrophy criteria, such as the Sokolow-Lyon, use only anterior and rightward forces for RV hypertrophy measurement and thus do not provide as accurate an estimation. Therefore, based on this reasoning, the $\mathrm{BL}$ criterion was chosen for testing its relationship to RV dilation.

Our study demonstrates a correlation between the BL criterion and $\mathrm{Qp} / \mathrm{Qs}$ ratio as a sign of $\mathrm{RV}$ volume overload on the ECG in patients with ostium secundum ASDs. It is worth noting that even after adjusting for PR interval and QRS duration, the $\mathrm{BL}$ criterion values were independently predictive of increasing intracardiac shunt ratios. Furthermore, the testing of different threshold values of the BL criterion for prediction of significant shunt ratios was deemed necessary because volume overload as opposed to pressure overload may have different effects on the ECG and this is a hitherto uninvestigated area.

The BL criterion is able to help determine a significant shunt ratio with a low threshold value of greater than $0 \mathrm{mV}$ possibly because of the fact that we are testing volume overload. It has been shown that pressure overloading of the heart affects the amplitude of the waves on the ECG. ${ }^{10}$ However, the ROC curve in Fig. 3 demonstrates that when compared with the RVH positive threshold value of greater than $0.7 \mathrm{mV}$, in volume overload a threshold value of greater than $0 \mathrm{mV}$ yields a higher sensitivity and specificity of 0.68 and 0.65 , respectively. Thus, in volume overload, we conclude that because of stretching of myocytes, ECG amplitude is less affected as compared with RV pressure overload. ${ }^{15}$

Our results indicate that in a population of adult patients with known ostium secundum ASDs, the ECG, a readily available and inexpensive test, may be useful in identifying those with significant intracardiac shunts. An ASD with a $\mathrm{Qp} / \mathrm{Qs}$ ratio greater than 1.5 is considered significant and warrants closure. ${ }^{16}$ Accordingly, using the BL criterion to determine a significant shunt ratio can be used in an outpatient clinic as a simple evaluation procedure to determine if the left-to-right shunt is significant. Although the positive predictive value is reasonable, the negative predictive value is low, indicating that a $\mathrm{BL}$ criterion value less than 0 may not be able to exclude a significant shunt. In other words, the value of a BL criterion greater than 0 in a patient with known secundum ASD carries more information for the treating clinician than a BL criterion value less than 0 .

It was shown in a previous study that the ECG shows prolongation of the PR interval in ostium secundum ASDs. ${ }^{8}$ Interestingly, we saw a similar relationship in our patient population. This is likely due to the rising $\mathrm{Qp} / \mathrm{Qs}$ ratio with the RA being subjected to a higher volume, consequent right atrial dilation, and thus increased duration for the electrical current to reach the His bundle. ${ }^{17}$

Moreover, recent literature suggests that ASDs are classified according to size of the defect and/or right-sided volume overload. ${ }^{18}$ Once again, the BL criterion can play a role in determining if there is significant right-sided volume overload. Although other noninvasive procedures for determining the $\mathrm{Qp} / \mathrm{Qs}$ ratio such as phase contrast cine MRI (with a sensitivity and specificity of 0.93 and 1 , respectively) are available, the lack of such facilities at many centers warrants investigation into the ECG as a simple procedure to identify those with a significant shunt ratio warranting ASD closure. ${ }^{19}$

Taking more direct indicators of RV volume overload, such as RV volume, and testing a relationship with the BL criterion could be an aim of a future study. In addition, because there are no specific ECG criteria for examining RV volume overload, future studies can possibly examine new ECG criteria specifically designed for RV volume overload. For example, because the volume overload affects the interventricular septum as well as the free RV wall, in some cases leading to paradoxical septal motion, it is possible that falsely positive ECG criteria for septal myocardial infarction might occur. These changes on ECG may need further investigation. Finally, a study with the follow-up BL criterion values of patients with closed ASDs at 6- and 12month intervals would further validate the value of using the $\mathrm{BL}$ criterion to assess RV volume overload.

\section{Study limitations}

Demographic, catheterization, and ASD data were electronically extracted from the DISCC database at Duke University Medical Center. Because all these data were put into our study database directly, the information was not verified patient-by-patient and thus could contain random errors. However, quality control was performed; and we checked for extreme values in the database (AMS). In addition, because of the retrospective nature of the study, there was no way of ensuring that all patients had progressed to the same stage in the natural history of the ASD; that is, volume overload for a prolonged time could have caused compensatory changes related to pressure overload. An analysis done to assess the natural history of the disease during diagnosis of the ASD would allow for all patients to be assessed based on their disease stage. Finally, some 
patients may have had tricuspid or pulmonic regurgitation, information which was not included in the database. These entities could raise the BL criterion values while maintaining low Qp/Qs ratios; and ideally, these patients should have been excluded from the analysis.

\section{Conclusion}

Our results indicate that there is limited utility of the BL criterion at detecting RV volume overload, although a $\mathrm{BL}$ criterion value greater than $0 \mathrm{mV}$ being used to identify patients with significant intracardiac shunts yielded a sensitivity of 0.68 and specificity of 0.65 . Thus, additional ECG criteria need to be developed to detect and estimate the magnitude of RV volume overload.

\section{Acknowledgments}

We would like to thank Phyllis Sewell, Sharon Sellers, Kenya Worley-Rankins, and Rieta Scarlett at the Duke University Medical Center Heart Station for their help in printing patient ECGs. In addition, we would like to thank Patricia Blunden-Kasdorf and Charles Trautman for their help in electronically extracting information from the DISCC database at Duke University Medical Center.

\section{References}

1. Cardoso CO, Rossi Filho RI, Machado PR, et al. Effectiveness of the Amplatzer device for transcatheter closure of an ostium secundum atrial septal defect. Arq Bras Cardiol 2007;88:384.

2. Gatzoulis MA, Redington AN, Somerville J, et al. Should atrial septal defects in adults be closed? Ann Thorac Surg 1996;61:657.

3. Brochu MC, Baril JF, Dore A, et al. Improvement in exercise capacity in asymptomatic and mildly symptomatic adults after atrial septal defect percutaneous closure. Circulation 2002;106:1821.

4. Thomson L, Crowley AL, Heitner JF, et al. Direct en face imaging of secundum atrial septal defects by velocity-encoded cardiovascular magnetic resonance in patients evaluated for possible transcatheter closure. Circ Cardiovasc Imaging 2008;1:31.
5. Piaw CS, Kiam OT, Rapaee A, et al. Use of non-invasive phase contrast magnetic resonance imaging for estimation of atrial septal defect size and morphology: a comparison with transesophageal echo. Cardiovasc Intervent Radiol 2006;29:230.

6. Ohlow MA, Secknus MA, von Korn H, et al. Incidence and outcome of femoral vascular complications among 18,165 patients undergoing cardiac catheterisation. Int J Cardiol 2008 [Electronic publication ahead of print].

7. Dill T. Contraindications to magnetic resonance imaging: non-invasive imaging. Heart 2008;94:943.

8. Madiyono B, Oesman IN, Sastroasmoro S, et al. Secundum atrial septal defect before and after surgery. Paediatr Indones 1989;29:199.

9. Butler PM, Leggett SI, Howe CM, et al. Identification of electrocardiographic criteria for diagnosis of right ventricular hypertrophy due to mitral stenosis. Am J Cardiol 1986;57:639.

10. Cabrera E, Monroy JR. Systolic and diastolic loading of the heart. II. Electrocardiographic data. Am Heart J 1952;43:669.

11. Wagner GS. Intraventricular conduction abnormalities. In: Wagner GS, editor. Marriott's Practical Electrocardiography. 11th ed. Philadelphia: Lippincott, Williams \& Wilkins; 2008. p. 104.

12. La Rosée K, Krause D, Becker M, et al. Transcatheter closure of atrial septal defects in adults. Practicality and safety of four different closure systems used in 102 patients. Dtsch Med Wochenschr 2001; 126:1030.

13. Jones TK, Latson LA, Zahn E, et al. Results of the U.S. multicenter pivotal study of the HELEX septal occluder for percutaneous closure of secundum atrial septal defects. J Am Coll Cardiol 2007;49:2215.

14. Di Bernardo S, Berger F, Fasnacht $M$, et al. Impact of right ventricular size on ECG after percutaneous closure of atrial septal defect with Amplatzer Septal Occluder. Swiss Med Wkly 2005;135:647.

15. Wiegerinck RF, Verkerk AO, Belterman $\mathrm{CN}$, et al. Larger cell size in rabbits with heart failure increases myocardial conduction velocity and QRS duration. Circulation 2006;113:806.

16. Arrington CB, Tani LY, Minich LL, et al. An assessment of the electrocardiogram as a screening test for large atrial septal defects in children. J Electrocardiol 2007;40:484.

17. Ho TF, Chia EL, Yip WC, et al. Analysis of $\mathrm{P}$ wave and $\mathrm{P}$ dispersion in children with secundum atrial septal defect. Ann Noninvasive Electrocardiol 2001;6:305.

18. Kharouf R, Luxenberg DM, Khalid O, et al. Atrial septal defect: spectrum of care. Pediatr Cardiol 2008;29:271.

19. Debl K, Djavidani B, Buchner S, et al. Quantification of left-to-right shunting in adult congenital heart disease: phase-contrast cine MRI compared with invasive oximetry. Br J Radiol 2009;82:386 [Electronic publication ahead of print]. 